\title{
Topographic Organization of Respiratory Responses to Glutamate Microstimulation of the Parabrachial Nucleus in the Rat
}

\author{
Nancy L. Chamberlin and Clifford B. Saper \\ Department of Neurology, Beth Israel Hospital, Harvard Medical School, Boston, Massachusetts
}

The parabrachial complex, also known as the pneumotaxic center or pontine respiratory group, has long been recognized as an important participant in respiratory control. One line of evidence supporting this idea is the demonstration of changes in breathing pattern following injection of neuroactive substances into or near the parabrachial complex. However, it is not yet known exactly which cell groups and projections mediate those responses. In order to address this issue, we explored the topographic organization of respiratory responses to chemical stimulation of the parabrachial complex of the rat and examined the descending projections of the most sensitive sites. Injection of glutamate (5-100 pmol) at specific sites in or near the parabrachial nucleus produced three distinct site-specific response patterns. First, hyperpnea followed glutamate injection into far rostral and midcaudal areas of the Kölliker-Fuse nucleus and most of the lateral parabrachial nucleus, including the external lateral, central lateral, dorsal lateral, and superior lateral subnuclei. Threshold hyperpneic effects were manifested as single, deepened breaths of premature onset. Suprathreshold doses of glutamate at these locations produced tachypnea. Neurons in these sites projected to the ventral respiratory group in the ventrolateral medulla. Second, the most intense inspiratory facilitatory responses were seen at mid to rostral levels of the Kölliker-Fuse nucleus, near the ventrolateral tip of the superior cerebellar peduncle. Even at threshold doses of glutamate, exhalation was incomplete, resulting in a breathing pattern that resembled apneusis (an inspiratory cramp). This site contained an especially dense cluster of neurons that projected either to the ventrolateral medulla or to the dorsal respiratory group in the nucleus of the solitary tract, but not to both areas. The third type of response, decreases in respiratory rate, occurred following glutamate injection at the most lateral and ventral boundaries of the Kölliker-Fuse nucleus. The most sensitive apneic sites were not found in the parabrachial nucleus but along the dorsal and medial edge of the principal sensory trigeminal nucleus and extending ventrally between the sensory and motor trigeminal nuclei. Scattered neurons in these sites were retrogradely labeled from the ventral but

\footnotetext{
Received Dec. 29, 1994; revised Apr. 7, 1994; accepted Apr. 21, 1994.

We thank Quan Hue Ha and Erica Sohl for excellent technical and administrative support, and Jon Sherin for helpful comments on the manuscript. This work was supported by NS22835.

Correspondence should be addressed to Dr. Nancy L. Chamberlin, Departmen of Neurology, Harvard Medical School, Building B1 room 153, 220 Longwood Avenue, Boston, MA 02115 .

Copyright (c) 1994 Society for Neuroscience $0270-6474 / 94 / 146500-11 \$ 05.00 / 0$
}

not the dorsal respiratory group. These results indicate that there are anatomically and functionally distinct cell populations in and near the parabrachial complex that, when chemically stimulated, can produce specific and sometimes opposing effects on respiration. The predominant effect of lateral parabrachial stimulation is respiratory facilitation, while inhibitory effects are elicited by trigeminal injections of glutamate.

[Key words: Kölliker-Fuse nucleus, respiration, microstimulation, trigeminal, apnea, apneusis, glutamate]

The parabrachial complex (PB), including the Kölliker-Fuse nucleus, is part of a key respiratory modulatory center known as the pontine respiratory group (see Feldman, 1986, for review). Early investigators found that lesions of the $\mathrm{PB}$ could produce apneusis or inspiratory cramps, leading them to propose that the PB functions as an important inspiratory off switch (Lumsdem, 1923). Indeed, some electrical stimulation experiments confirmed that activation of neural elements in the medial PB or the Kölliker-Fuse nucleus (KF) caused shortening of inspiration and lengthening of the expiratory phase, which, at the extreme, produced apnea (Cohen, 1971). However, stimulation of the lateral PB revealed facilitatory effects of parabrachial neurons on inspiration, findings not supportive of the idea that the PB functions solely as an inspiratory off switch. For example, electrical or chemical stimuli delivered at certain sites in the PB during expiration caused early initiation of inspiration and an increase in the amplitude of phrenic nerve discharge (Baxter and Olszewski, 1955; Bertrand and Hugelin, 1971; Cohen, 1971; McCrimmon et al., 1986; Miura and Takayama, 1991). The apparent inconsistencies in the reported effects of PB stimulation on breathing could be accounted for by the existence of separate groups of cells within or near the PB with different and possibly opposing physiological roles in respiration. However, this hypothesis has not yet been tested for two reasons. First, the magnitude of electrical currents and doses of chemoexcitants used in previous studies were too large to selectively stimulate small subsets of parabrachial neurons. Second, these experiments were done using cats or other species in which the parabrachial subnuclei and their connections have not yet been defined in detail (and might not be spatially distinct). Thus, in order to determine which pontine cell groups and connections were associated with the different respiratory responses, we stimulated the PB and surrounding area in the rat, a species in which the PB consists of distinct and well-characterized subnuclei, with small doses of glutamate and observed the effects on breathing. We further investigated the possible anatomical pathways through which the PB might influence breathing by 
comparing the locations of glutamate responsive sites with those of neurons that project to the medullary ventral and dorsal respiratory groups, in the ventrolateral medulla and the nucleus of the solitary tract, respectively.

We now report the anatomical localization of three different types of respiratory responses that resulted from chemical stimulation of different parts of the PB and surrounding area. These data dispute the hypothesis that the PB serves solely as an inspiratory off switch, suggesting instead a more complex interplay of $\mathrm{PB}$ influences on respiratory rhythm regulation.

A preliminary report of these studies has appeared (Chamberlin and Saper, 1990).

\section{Materials and Methods}

Seventy-five male Sprague-Dawley rats $(300-400 \mathrm{gm})$ were anesthetized with $\alpha$-chloralose (100-150 mg/kg, i.p.). Some rats also received a single preoperative injection of chloral hydrate $(200 \mathrm{mg} / \mathrm{kg}$, i.p.). Subsequently, injections of $\alpha$-chloralose ( $45 \mathrm{mg} / \mathrm{kg}$, i.v.) were given every $1-2 \mathrm{hr}$, to maintain an anesthetic state in which the rat did not respond to a noxious foot pinch and the mean arterial blood pressure was below $130 \mathrm{~mm}$ Hg. Rectal temperature was maintained at $37 \pm 2^{\circ} \mathrm{C}$ with a $75 \mathrm{~W}$ incandescent lamp. Polyethylene catheters were placed in a femoral artery and vein and into the trachea. The distal end of the endotracheal tube was attached to a Y-connector through which flowed humidified gas $(100 \%$ oxygen) at a rate of $100-500 \mathrm{ml} / \mathrm{min}$. This flow was monitored with a Fleisch pneumograph. The respiratory flow, blood pressure, and stimulus monitor signals were amplified by a Grass polygraph and recorded on 4-channel VCR tape (Vetter). These signals were digitized at $1.5 \mathrm{kHz}$ by a computer equipped with $\mathrm{A} / \mathrm{D}$ acquisition hardware and software (TL-2 or TL-1 and Axotape, Axon Instruments, Foster City, CA). Analysis and plots of binary data records were performed with an Apple Macintosh computer equipped with IRGo software (Wavemetrics, Lake Oswego, OR). Inspiratory and expiratory airflow was reflected as a decrease or increase, respectively, in the air flow measured by the Fleisch pneumograph. In order to determine tidal volume, a constant was subtracted from the airflow trace to zero the baseline, and the result was integrated. The system was calibrated with a $1 \mathrm{ml}$ syringe attached to the port of the Y-connector normally attached to the tracheal tube. Sometimes small changes in the flow of oxygen were observed as a slow drift in the baseline of the tidal volume signal (e.g., Fig. 4B). Rats were placed in a stereotaxic frame (Kopf) with the incisor bar set at interaural zero and a craniotomy was performed to allow access to the left PB.

Multibarrel micropipettes were used to inject glutamate. Triple barrel pipettes (outer diameter of tip assembly, 30-50 $\mu \mathrm{m}$ ) were constructed from nonheparinized hematocrit tubes (Clay Adams), and the different barrels were filled with L-glutamate ( $\mathrm{Na}$ salt, Sigma; $1-10 \mathrm{~mm}$, in 0.1 м Na phosphate buffer, pH 7.4), 0.1 м Na phosphate buffer $\mathrm{pH} 7.4$, and $2 \%$ Pontamine sky blue in $3 \mathrm{M} \mathrm{NaCl}$ and $4-5 \%$ biocytin (Sigma). The three solutions were used for chemical stimulation, as a control for pressure injection, and for electrical stimulation and dye injection, respectively. Double barrel pipettes were constructed from theta glass (World Precision Instruments). One side contained glutamate (1-10 mM) and the other contained $3 \mathrm{~m} \mathrm{NaCl}, 2 \%$ Pontamine sky blue, and $5 \%$ biocytin (Sigma).

The PB was explored with $10 \mathrm{sec}$ trains of negative current pulses (5$30 \mu \mathrm{A}, 0.4 \mathrm{msec}, 50 \mathrm{~Hz})$ and microinjections of L-glutamate $(5-50 \mathrm{nl}$ 1-10 $\mathrm{mm}$ ). To minimize tissue damage, the larger volumes were ejected in a series of small pressure pulses $(2-5 \mathrm{nl} / \mathrm{pulse})$. In the mapping experiments, these pulses were not timed to occur in any particular phase of the respiratory cycle. In 12 experiments, an equal volume of phosphate buffer was injected at the same site at which responses to glutamate were observed. Since no responses were seen after phosphate buffer injection, this control was omitted in later experiments. Biocytin was iontophoresed by a $+3-4 \mu \mathrm{A}$ current (continuous or $7 \mathrm{sec}$ on $/ 7 \mathrm{sec}$ off) for 20-45 min at the most ventral stimulation site in each pipette track. Rats were not allowed to recover from anesthesia following the experiment and 15-60 minutes following biocytin injection were perfused through the heart under deep anesthesia with saline $(0.9 \%)$ followed by formaldehyde ( $4 \%$ in $0.1 \mathrm{M} \mathrm{Na}$ phosphate buffer, $\mathrm{pH} 7.4$ ). Following fixation, the skulls were opened to expose the dorsal surface of the brain and mounted in a stereotaxic frame, and a single vertical cut was made with a scalpel blade through the brain rostral to but in the same plane

\section{A}
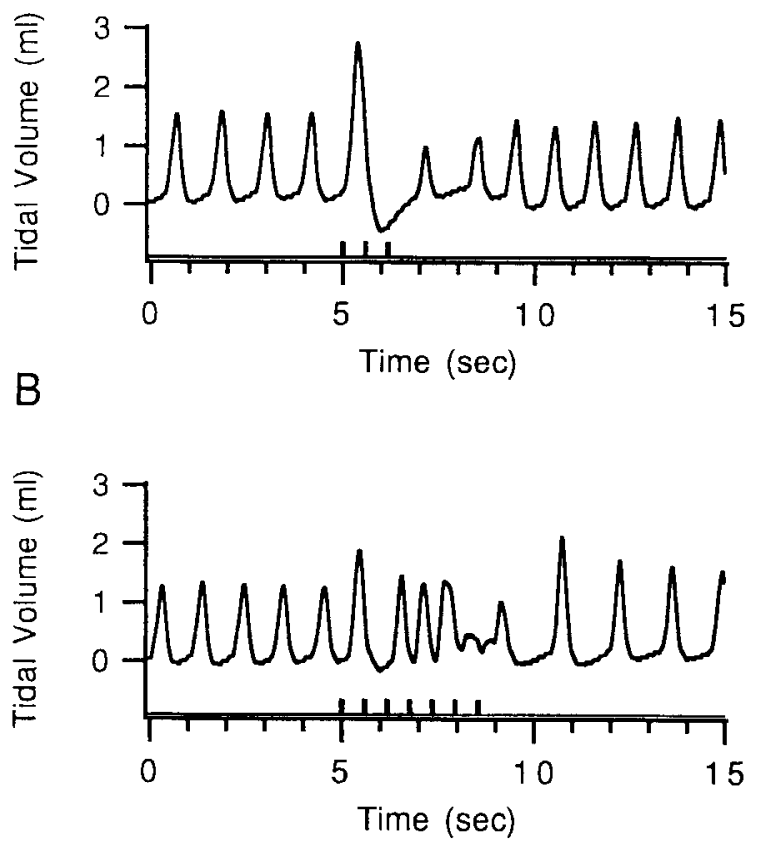

Figure 1. Hyperpneic responses following glutamate injection into the parabrachial nucleus. $A$ and $B$, Shown are changes in respiration in response to two different doses of glutamate injected at a single site in the PB (experiment RF644). The vertical deflections from the time axis denote the occurrence of glutamate pressure pulses (3-5 nl each). In $A$ the trace shows a brief threshold response to glutamate $(10 \mathrm{pmol}) . B$ shows that a higher dose of glutamate ( $30 \mathrm{pmol})$ caused tachypnea.

as the stimulation pipette track. Brains were removed and immersed overnight in sucrose ( $20 \%$ in phosphate buffer), and frozen sections were cut at $50 \mu \mathrm{m}$ through the PB of each brain in the same plane as the micropipette penetration. In order to visualize the biocytin injection sites, free-floating tissue sections were incubated in streptavidin conjugated to horseradish peroxidase $(1.7 \mu \mathrm{g} / \mathrm{ml}$ in phosphate-buffered saline, PBS; $\mathrm{pH} \mathrm{7.4)}$ or streptavidin conjugated to Texas red at room temperature for $1 \mathrm{hr}$. After rinsing in PBS, the tissue sections labeled with horseradish peroxidase were reacted with $0.05 \%$ diaminobenzidine and $0.01 \%$ hydrogen peroxide in PBS. All sections were mounted on gelatin-coated glass slides. Slide-mounted Texas red-labeled tissue was air dried and coverslipped with Permaslip. Slides with diaminobenzidine-labeled tissue were dehydrated in a graded series of ethanols, cleared in xylene, and coverslipped with Histoclad (Clay Adams), DPX (Gallard, Schlesinger, Chem., Carle Place, NY) or Permaslip (Newcome, Oak Park, IL).

The sites of diaminobenzidine-HRP reaction product labeling were mapped with respect to tissue landmarks on camera lucida drawings, while fluorescence-labeled tissue was mapped with a computerized stage position plotting system (MDPLOT, Minnesota Datametrics). All the tissue sections were subsequently counterstained with thionin. Using a camera lucida drawing tube, the positions of $\mathrm{PB}$ subnuclei were added to complete the drawings. Composite drawings of glutamate stimulation sites were made with a camera lucida drawing tube as previously described (Chamberlin and Saper, 1992).

In 32 experiments, 1-2 weeks prior to the physiological mapping experiments described above, rats injected with retrograde tracers. Briefly, rats were deeply anesthetized with $7 \%$ chloral hydrate (1.5-2 ml, i.p.) and mounted in a stereotaxic frame with the head in a ventroflexed position. Access to the medulla was obtained by incising the skin, reflecting the neck musculature from the occipital bone, and cutting open the overlying dura. Using the calamus scriptorius as a point of reference, stereotaxic injections of fast blue (2-5\% in saline, $25-30 \mathrm{nl})$ and diamido yellow $(2-5 \%, 30-60 \mathrm{nl})$ dyes were placed into two sites in the medulla: either the rostral and caudal portions of the ventrolateral medulla, or 
A

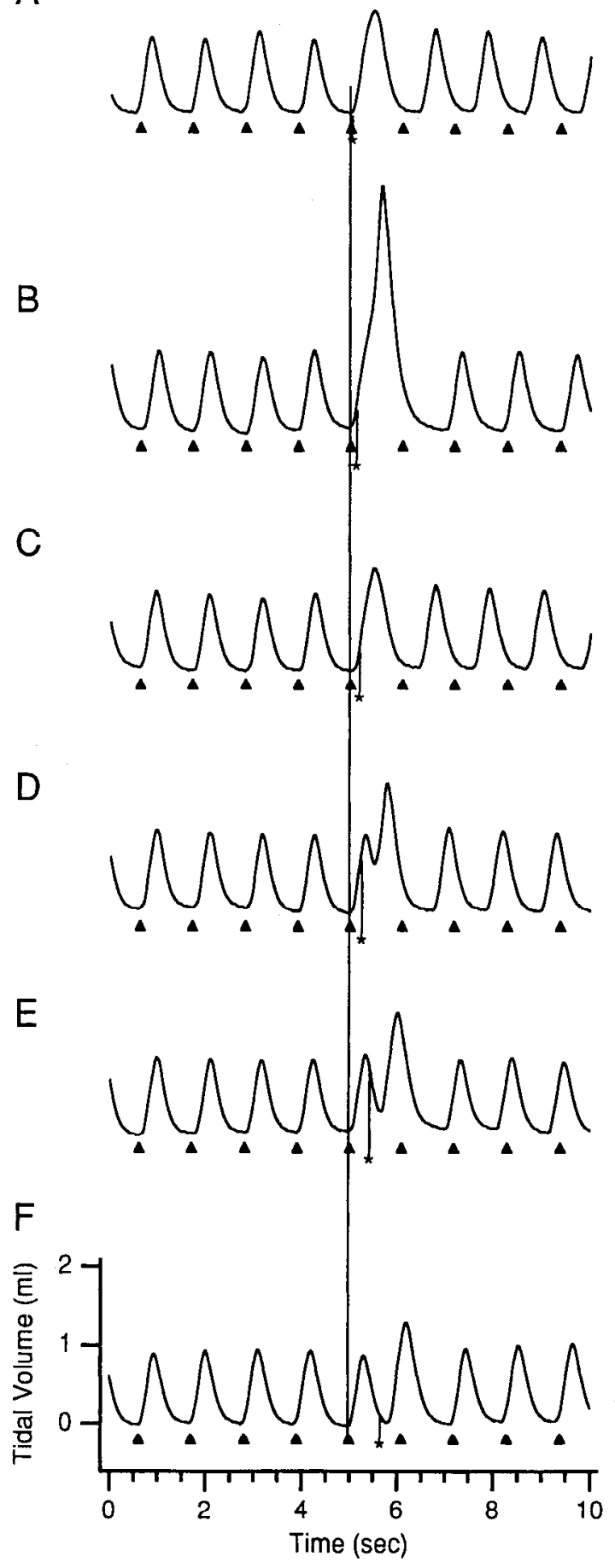

Figure 2. Influence of the respiratory phase on responses to glutamate $(5 \mathrm{nl}, 10 \mathrm{~mm}$ ) microinjection. $A-F$ show respiratory changes in response to six glutamate applications (asterisks) at the same site in one rat (case SPB441). Traces are lined up such that the breath just prior to glutamate administration occurs at the $5 \mathrm{sec}$ time point. The triangles below each trace denote the times of inspiratory initiation before glutamate administration. After glutamate application (asterisks), the triangles show the expected time of inspiratory onset. Note that the prestimulation respiratory rate ( 55 breaths $/ \mathrm{min}$ ) is the same in $A-F$ and that following stimulation the respiratory rhythm was reset.

the nucleus of the solitary tract in combination with one of the ventrolateral medullary sites. The skin incisions were closed with wound clips and the animals allowed to recover from anesthesia. After 1-2 weeks the rats were used for glutamate microstimulation experiments as de-
A

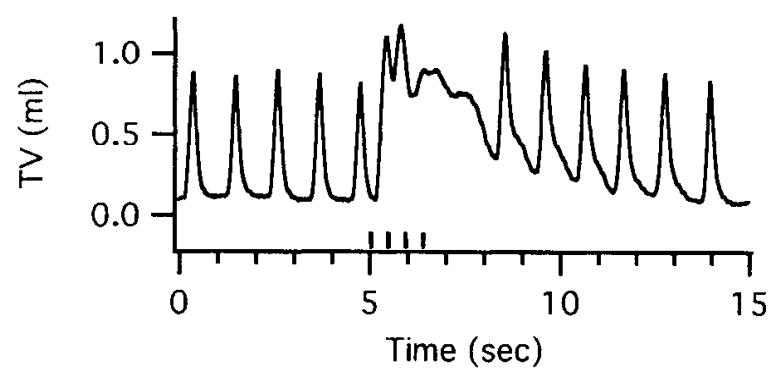

B

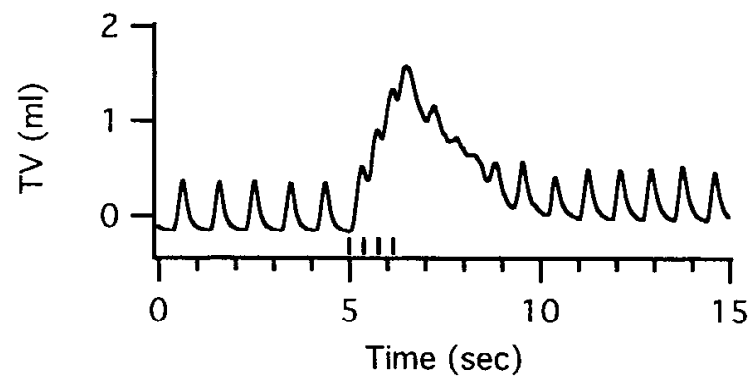

Figure 3. Apneustic responses following glutamate injection into the Kölliker-Fuse nucleus. $A$ shows the change in respiration after glutamate (100 pmol in four pulses) in case SPB459. $B$ shows the effect of glutamate ( $5 \mathrm{pmol}$ in four pulses) in another case (SPB488).

scribed above. In these cases, the distributions of retrogradely labeled cells were compared to the stimulation sites, and the relationship documented either photographically or with the MDPLOT computerized plotting system.

\section{Results}

Respiratory responses to glutamate injection into the $P B$

Three different types of respiratory responses were observed following injection of glutamate $(5-100 \mathrm{pmol})$ into the dorsolateral pons: brief increases in respiratory rate and tidal volume (hyperpnea) (Figs. 1, 2), apneusis (Fig. 3), and apnea (Fig. 4).

Hyperpneic responses were typically manifested as an increase in tidal volume and an advance in the time of onset of the first breath following glutamate injection (Figs. 1, 2). These brief responses were produced by amounts of glutamate as low as 510 pmol, whereas larger doses of glutamate $(>10 \mathrm{pmol})$ at the same sites caused tachypnea: two or more breaths with reduced interbreath intervals. Occasionally tachypnea was also observed at the lowest doses.

In order to determine the influence of the timing of glutamate application on the hyperpneic responses, repeated injections were made at a single site (Fig. 2). We found that varying degrees of tidal volume enhancement and advancement of the time of inspiratory onset could be accounted for by the timing of glutamate application with respect to the respiratory cycle. As illustrated by the case shown in Figure 2, glutamate injection early in inspiration tended to increase the volume of a single breath (Fig. $2 A-C$ ). When glutamate was delivered later in inspiration or during expiration (presumably too late to affect the ongoing breath), the time of inspiratory onset of the following breath was advanced and the volume was increased (Fig. 2D$F)$. The interval between the augmented, premature breath and the subsequent breath was often lengthened. The resulting change in interbreath interval caused a resetting of the respiratory rhythm 
(Fig. 2). The effect of the timing of glutamate application on the amplitude of the first breath following injection is also apparent in Figure 1 (compare $A$ and $B$ ).

Apneusis was produced by amounts of glutamate as low as 5 pmol (Fig. 3). This response was qualitatively different from tachypnea in the sense that inspiration time was greatly prolonged and lung volume did not return to baseline between breaths. This breathing pattern might have been produced by long, uninterrupted phrenic bursts, by an increase in respiratory rate such that there was insufficient time for complete exhalation between breaths, or by a combination of increased inspiration time and decreased expiration time. Apneustic responses were followed by a normal, but reset respiratory rhythm.

The third type of response that occurred following glutamate administration into sites within or near the parabrachial complex was a decrease in respiratory rate, tidal volume, or both (hypopnea) (Fig. 4). The salient feature of the hypopneic response was an increase in expiratory time seen as a delay in the time of onset of the first breath that followed glutamate application. The duration of these respiratory pauses was dose dependent; 100 pmol glutamate could cause periods of apnea exceeding $10 \mathrm{sec}$ (Fig. 4). Breathing usually resumed at a reduced tidal volume but returned to control levels within a few breaths, and the rhythm was reset. Unlike the hyperpneic response, the nature of the hypopneic response did not appear to be dependent upon the time of glutamate injection with respect to the respiratory cycle; that is, the last breath prior to the apnea invariably occurred with the proper timing and duration.

\section{Blood pressure responses to glutamate injection into $P B$}

Blood pressure changes were very small in these experiments ( $<10 \mathrm{~mm} \mathrm{Hg}$ ), consistent with previous observations that the doses of glutamate used in this study (generally $<25$ pmol) are barely enough to change blood pressure (Chamberlin and Saper, 1992). In fact, most of the changes in blood pressure that were observed in these experiments appcarcd to be secondary to changes in breathing due to heart rate fluctuations in phase with respiration (respiratory sinus arrhythmia).

The abrupt, premature inspiration triggered by glutamate at most hyperpneic sites was often accompanied by small, biphasic (increase followed by decrease) blood pressure changes lasting 3-7 sec. Blood pressure changes during apneusis were inconsistent; small increases, decreases, or biphasic (increase followed by decrease) effects were observed. Slight decreases in blood pressure occurred during most hypopneic responses.

\section{Anatomical distribution of respiratory modulatory sites}

The anatomical locations at which glutamate injection caused the three types of respiratory responses is shown in Figure 5. Prolonged increases in inspiratory duration were produced when glutamate was injected into a rostral region of the Kölliker-Fuse nuclcus. These apncustic sites were clustered ventrolateral to the superior cerebellar peduncle at this one level (Fig. 5A,B).

In contrast, respiratory facilitatory effects that consisted of a single augmented breath or transient tachypnea occurred following glutamate application into several subnuclei in the lateral PB. These areas included the superior lateral, lateral crescent (see Chamberlin and Saper, 1992), dorsal lateral, external lateral, and central lateral subnuclei, the more caudal KöllikerFuse nucleus, and the area just medial to the Kölliker-Fuse nucleus (Fig. 5).

Glutamate, injected into the farthest lateral and ventral as-
A

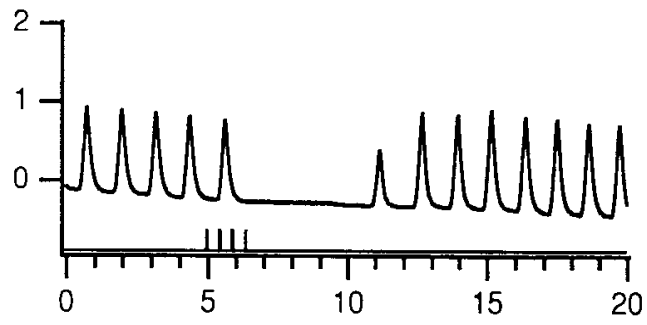

B

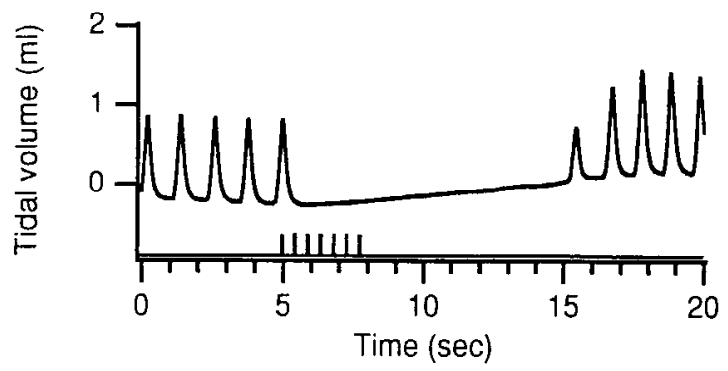

Figure 4. Apnea following glutamate injection into the PB. $A$ shows an increase in expiratory duration following injection of glutamate (50 pmol) in case SPB458. $B$ shows the effect of a higher dose of glutamate $(100 \mathrm{pmol})$ at the same site. The baseline drift during the apneic interval in $B$ is artifactual.

pects of the Kölliker-Fuse nucleus, caused apnea (Fig. 5). Decreases of respiratory rate were also elicited from sites in the principal sensory trigeminal nucleus; the most sensitive sites were along the dorsal and medial edges of this nucleus and extending into the cells and fiber bundles that lie between the scnsory and motor trigcminal nuclei (Fig. 5). No respiratory changes followed glutamate injection into the motor trigeminal nucleus, although occasional jaw or whisker movements were observed.

\section{Comparison of respiratory sites to the location of neurons retrogradely labeled from medullary respiratory groups}

In order to determine which parabrachial projection neurons were potential candidates for mediating the observed changes in respiration, we compared the pontine respiratory responsive sites with the locations of subpopulations of neurons selectively labeled by injections of the fluorescent retrograde tracers fast blue and diamido yellow into their medullary terminal fields. Neurons labeled with these two dyes could be distinguished not only by their color, but also because diamido yellow only labels nuclei while fast blue also stains cytoplasm (Fig. 6). The injection sites were chosen based on their known involvement in respiratory control: the ventrolateral medulla and the nucleus of the solitary tract. Two sets of experiments were done.

First, in 15 experiments, one injection of diamido yellow was placed into the rostral ventrolateral medulla at the level of the compact formation of the nucleus ambiguous just rostral to the level at which the nucleus of the solitary tract touches the floor of the fourth ventricle (the level of the Bötzinger complex) and an second injection of fast blue was placed into the caudal ventrolateral medulla, usually just below the level of the obex. The pattern of labeling was similar in each case to that previously 

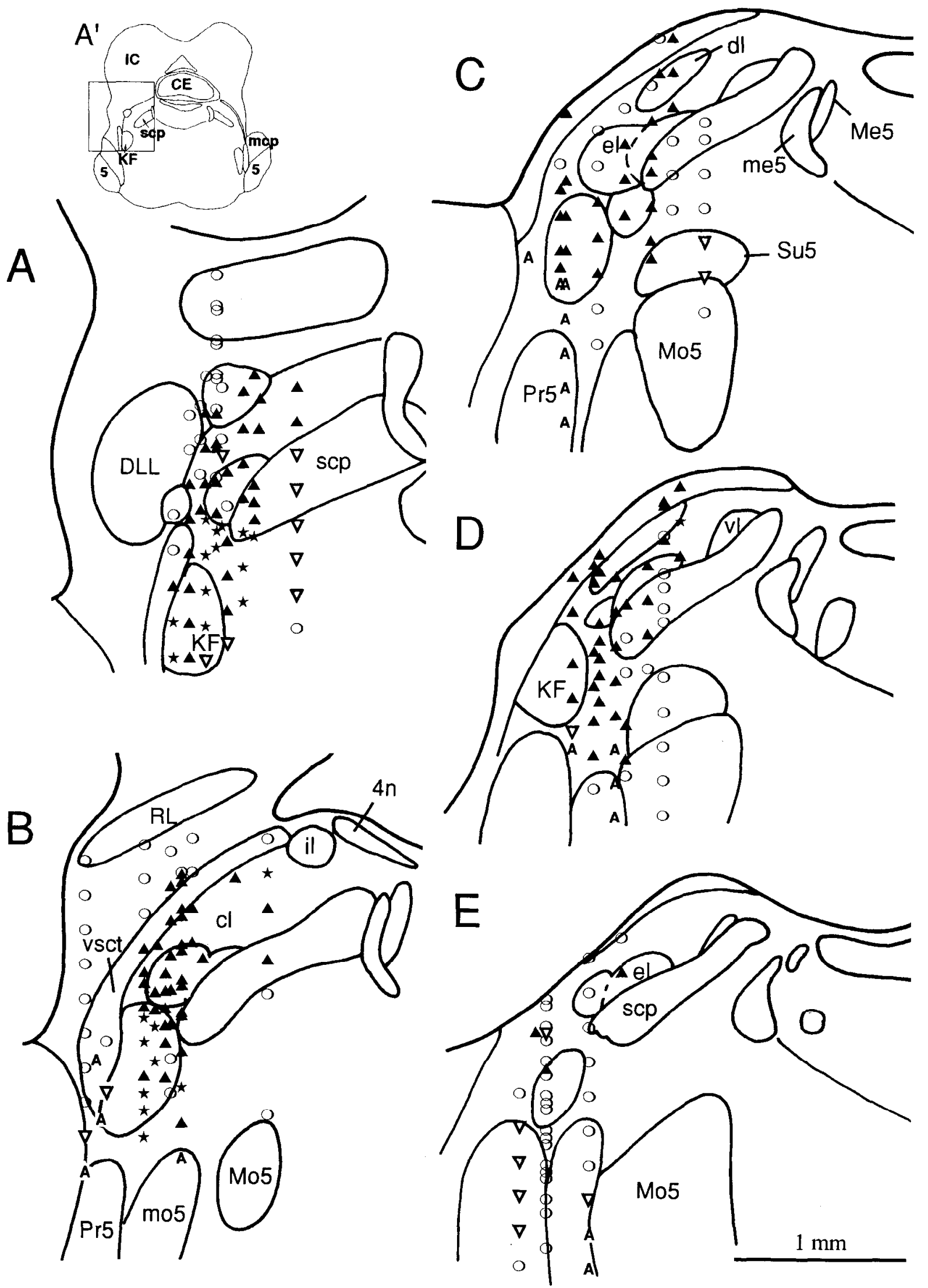

Figure 5. Anatomical distribution of respiratory responses to glutamate microinjection into $\mathrm{PB}$. $A-E$ are composite drawings of five levels of the $\mathrm{PB}$ (rostral to caudal). The inset $(A)$ is a drawing of the most rostral representative section. Each symbol represents the type of respiratory response at a single site where glutamate ( $10 \mathrm{nl}, 1-10 \mathrm{~mm}$ ) was injected. Open circles, no response; stars, apneusis; solid triangles, incrcased rate (or volumc); 
described by Herbert et al. (1990). The distributions of neurons that were retrogradely labeled by the two injection sites were indistinguishable; in fact, up to $15 \%$ of the retrogradely neurons in the KF and lateral PB in these experiments were double labeled.

Glutamate microstimulation experiments were conducted on a second set of 17 rats that had fast blue dye placed into the nucleus of the solitary tract at or just caudal to the level of the area postrema and diamido yellow injected into either the rostral or caudal ventrolateral medulla. In these experiments, the bulk of the neurons retrogradely labeled from the nucleus of the solitary tract were confined to the KF in exactly the same region in which glutamate produced the apneustic responses (see Fig. 7). There were very few double-labeled neurons, although the diamido yellow- and fast blue-labeled neurons were thoroughly intermixed.

\section{Discussion}

This study describes functionally and anatomically distinct subregions of the parabrachial complex that affect breathing in the rat. We found three different patterns of respiratory responses, hyperpnea, apneusis, and apnea, each associated with a different anatomical location and distinct pattern of descending projections. As the most prevalent response that followed glutamate application into the PB was brisk respiratory facilitation in the form of hyperpnea or apneusis, it appears that the major influence of the PB is to promote lung inflation (Figs. 1-3, 5, 7). In contrast, inspiratory inhibitory effects only occurred following glutamate injections into the ventral rim of the PB and into the trigeminal complex, suggesting that the neurons that constitute the pontine inspiratory off switch hypothesized to reside in the PB complex instead are located just ventral to the KF.

\section{Technical limitations}

The experiments discussed in this report were all done on spontaneously breathing, vagally intact rats. Thus, ventilatory changes were likely to have been tempered by respiratory reflexes, potentially making it more difficult to observe responses to microstimulation. This potential problem could have been avoided by using vagotomized, paralyzed and ventilated rats. In the absence of the pulmonary stretch information conveyed by the vagus nerve and the changes in blood gas levels concomitant with changes in ventilation, the effect of brain microstimulation on phrenic output is more easily observed. However, the profuse reciprocal relationship of the $\mathrm{PB}$ with not only the respiratory pattern generating and premotor neurons in the ventrolateral medulla, but also the chemosensory and pulmonary stretch receptive regions of the nucleus of the solitary tract suggests that the actions of the PB might be integrated into the sensory as well as the motor limb of the central respiratory control network. If so, the absence of sensory feedback might eliminate some or all of the physiologically relevant effects of PB stimulation. Therefore, we chose not to deafferent, paralyze, and ventilate rats in our experiments.

We decided to record respiratory flow, as it is a more direct

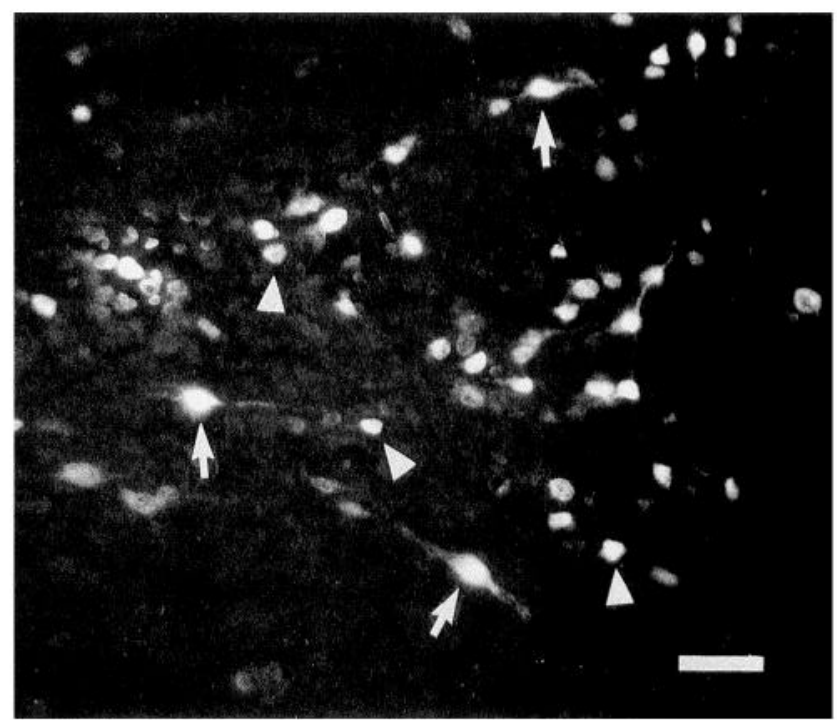

Figure 6. Appearance of fast blue- and diamido yellow-labeled neurons: high-magnification photomicrograph of retrogradely labeled parabrachial neurons following an injection of fast blue into the NTS and diamido yellow into the rostral ventrolateral medulla of one rat. Note that fast blue labeled the cell bodies and proximal dendrites (arrows), whereas diamido yellow only labeled nuclei (arrowheads). Scale bar, 40 $\mu \mathrm{m}$.

measurement of lung ventilation, rather than phrenic nerve activity. However, under these recording conditions it was not possible to determine whether the neural mechanism underlying apneusis was a prolonged, uninterrupted phrenic nerve burst or a run of tachypneic breaths in which the expiratory phase was neurologically normal, but too short to allow for complete exhalation between breaths. Hence, although we refer to these responses as apneusis, and they were produced at threshold doses of glutamate, it is possible that they were simply extreme forms of tachypnea. In future studies phrenic nerve recordings would allow a distinction to be made between these alternative mechanisms.

Since a major objective of this study was to localize physiological responses to glutamate stimulation with respect to particular groups of neurons, it is well to consider factors that may determine which neurons are activated by glutamate microinjections. One consideration is the possibility of inhibition of the cells nearest the pipette tip due to excessive depolarization (Lipski et al., 1988). However, we do not feel that this contributed to the respiratory responses in our study since we used very small volumes and low concentrations of glutamate, amounts 1000 -fold lower than those shown to produce depolarization block (Lipski et al., 1988). Another potential problem that might be encountered is excitotoxicity; application of supraphysiological doses of glutamate that overwhelm endogenous uptake and clearance processes could potentially harm, or even kill the neurons. This phenomenon would not affect the results of our study since we were concerned only with the immediate effects

inverted open triangles, decreased rate but not doubling of interbreath interval; $A$, apnea (interbreath interval $\geq 2 \times$ control). Apneic responses also were observed at sites ventral to regions shown. $d l$, dorsal lateral PB subnucleus; $D L L$, dorsal nucleus of the lateral lemniscus; $e l$, external lateral PB subnucleus; $i l$, internal lateral PB subnucleus; $K F$, Kölliker-Fuse nucleus; $M e 5$, mesencephalic trigeminal nucleus; me5, mesencephalic trigeminal tract; $M o 5$, motor trigeminal nucleus; $m o 5$, motor trigeminal tract; $\operatorname{Pr} 5$, principal sensory trigeminal nucleus; $R L$, retrolemniscal nucleus; $s c p$, superior cerebellar peduncle; $S u 5$, supratrigeminal nucleus; $v l$, ventrolateral PB subnucleus; $v s c t$, ventral spinocerebellar tract; $4 n$, trochlear nerve. 
C
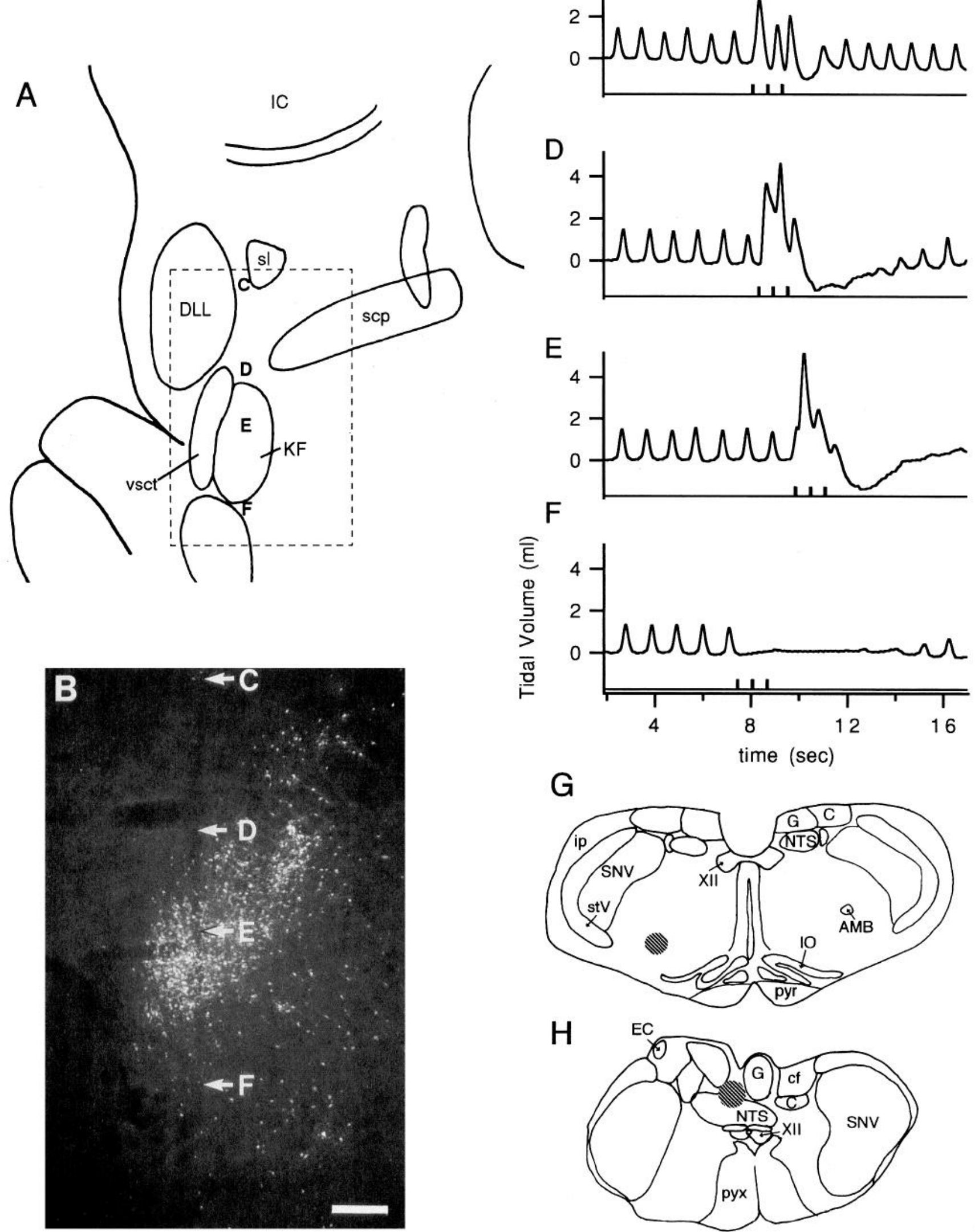

G

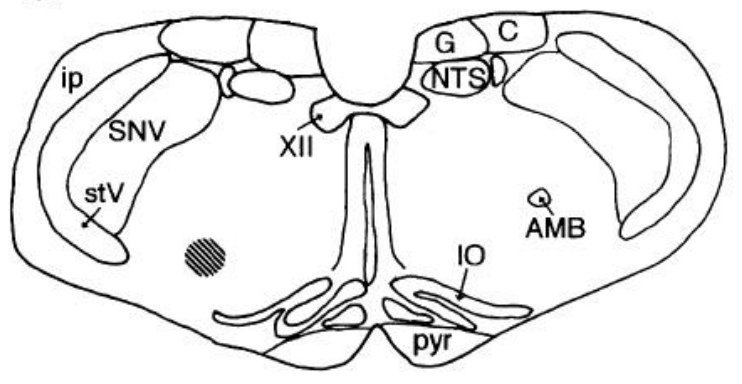

H

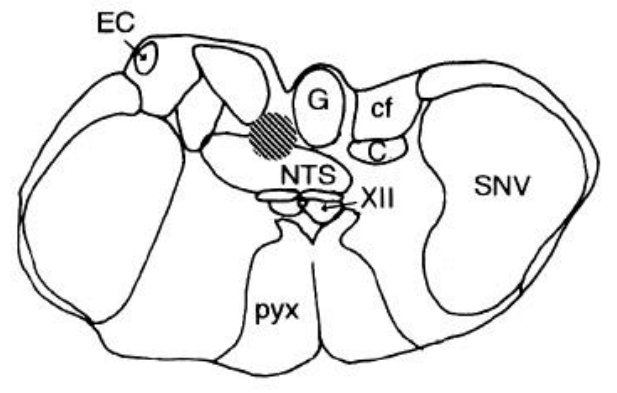


of glutamate injection; whether the cells would have died later if the animals survived does not affect our interpretation of the results. Finally, an unavoidable limitation of glutamate microstimulation that bears direct relevance to this study is the potential dendritic activation of neurons with cell bodies distant from the injection site. The subnuclear arrangement of the dendritic trees of parabrachial neurons is unknown. This is unfortunate, since such information would be a valued aid in the interpretation of microinjection studies.

\section{Respiratory responses to $P B$ microstimulation}

Our results confirm the existence of respiratory responses found by prior microstimulation studies of the PB and further extend our knowledge to include additional responses not previously reported and their topographic organization with respect to parabrachial subnuclei. Early studies of paralyzed, ventilated cats showed that electrical stimulation of the dorsal PB induced the onset of and increased the amplitude of phrenic nerve activity (Baxter and Olszewski, 1955; Cohen, 1971; Baker and Remmers, 1982; Bassal and Bianchi, 1982), whereas phrenic nerve discharges were cut short by stimuli at more ventral sites (Bertrand and Hugelin, 1971; Cohen, 1971; Euler and Trippenbach, 1976; Baker and Remmers, 1982). Similar effects of electrical stimuli were observed in paralyzed, ventilated rats (Wang et al., 1993). However, it was not clear in these studies whether some of the responses were due to activation of fibers of passage originating from cells outside of rather than within the PB.

Chemical parabrachial stimulation in paralyzed, ventilated cats has been reported to increase the amplitude and frequency of phrenic nerve discharge and in spontaneously breathing cats to increase tidal volume and respiratory rate (McCrimmon et al., 1986; Miura and Takayama, 1991; Takayama and Miura, 1993). In studies where blood pressure was recorded in cats it was shown that the threshold dose of glutamate required to raise blood pressure was greater than the amounts that were necessary to affect breathing. Our results showed this also to be true in the rat. The existence of chemically stimulated hypopnea from the KF in rats has been suggested in preliminary reports (Fournier and Feldman, 1988; Chamberlin and Saper, 1990), but this is the first systematic study that has attempted to localize this response. Our results indicate that hypopnea is mediated primarily by cells near, but outside of the KF.

Two of the three different chemically elicited response patterns that we observed in the parabrachial region of the rat, hypopnea and apneusis, have not been described in similar studies in the cat, perhaps because apneustic and respiratory depressant sites, which are more ventrally located than most hyperpneic sites in the rat, were not stimulated in those experiments. The absence of reported apneustic effects of glutamate injections in cats might also be explained if the pontine glutamate injec- tions in our experiments caused apneusis by reducing the expiratory period to a time insufficient for complete exhalation. As cats have a longer respiratory cycle than rats, they might be less susceptible to apneusis because they have a proportionately longer opportunity to exhale. Finally, it is conceivable that pontine respiratory control is fundamentally different in rats and cats. However, given the paucity of knowledge concerning the organization of the PB in the cat, it is difficult to reach any firm conclusions regarding physiological mechanisms by comparing effects of microstimulation in the two species.

\section{$P B$ synaptic targets subserving respiratory responses}

The three types of respiratory responses that we observed in the PB originated from sites having different patterns of efferent projections.

Hyperpneic responses were obtained from several PB subnuclei, each with a unique set of projections (Fulwiler and Saper, 1984). Although the hyperpneic sites project to a number of different nuclei in the basal forebrain, they all contain neurons that project to the ventrolateral medulla (Herbert et al., 1990; Chamberlin and Saper, 1992). The common descending projection to the ventrolateral medulla, taken together with the known role of this region in respiratory control, suggests that this latter pathway probably underlies the hyperpneic response.

An exception is the respiratory facilitation obtained by stimulating the external lateral subnucleus, which is singularly devoid of labeled neurons following injections of retrograde tracers into the medulla. However, the hyperpneic responses tended to cluster along the margins of the external lateral subnucleus from which glutamate may have spread into the adjacent lateral crescent area, which does project to the ventrolateral medulla. It is also possible that forebrain targets of the external lateral cell group might participate in causing hyperpnea. Indeed, electrical stimulation of one of these targets, the central nucleus of the amygdala, has been shown not only to trigger inspiration, but also to entrain respiration (Ilarper et al., 1984).

The neurons of the ventral respiratory group, which has been hypothesized to generate the respiratory pattern, determine the breathing rate, and drive the phrenic and intercostal motor neurons (Smith et al., 1991), lie in a column that occupies the ventrolateral quadrant of the medulla from just caudal to the facial motor nucleus to the medullospinal junction. This entire region is richly innervated by axons originating from cells in the PB (Herbert et al., 1990; Chamberlin and Saper, 1992). However, it is not known which subsets of neurons are contacted by parabrachial axons. The ventrolateral medulla contains at least three intermingled populations of neurons that participate in respiratory control: premotor neurons that project to the spinal cord, interneurons that form connections within the medulla and probably generate the respiratory rhythm, and vagal

\footnotetext{
Figure 7. Relationship between the respiratory responses to glutamate and the locations of neurons that project to the rostral ventrolateral medulla and caudal commissural subnucleus of the solitary tract. Respiratory responses following glutamate injection (10 nl, $1 \mathrm{~mm}$ ) at four sites are shown. $A$, Camera lucida drawing of a rostral level of PB depicting the spatial relationship between the locations of physiological responses to glutamate denoted by the letters $C-F$ and the parabrachial subnuclei. $B$, Photomicrograph of the same section showing diamido yellow-labeled nuclei and fast blue-labeled cells following injections of these dyes into the rostral ventrolateral medulla and nucleus of the solitary tract, respectively. Note the widespread distribution of diamido yellow-labeled nuclei with the highest density ventrolateral to the superior cerebellar peduncle. Scale bar, $200 \mu \mathrm{m}$. The four arrows in $B$ denote the locations of the respiratory responses that are shown in traces $C-F$. Stimulation at the most dorsal site $(C)$, whose neurons were retrogradely labeled only from the ventrolateral medulla, caused tachypnea, whereas apneustic effects $(D$ and $E)$ were closely associated with a cluster of neurons in the rostral KF that were retrogradely labeled either from the ventrolateral medulla or from the nucleus of the solitary tract. In contrast, hypopnea or apnea $(F)$ was only obtained following glutamate injections ventral to the KF, where scattered neurons projecting to the ventrolateral medulla were found. $G$ and $H$ show the sites of diamido yellow $(G)$ and fast blue $(H)$ injections.
} 
motoneurons (Ellenberger and Feldman, 1990). The ventrolateral medulla also contains sympathetic premotor neurons and interneurons involved in cardiovascular regulation. At the rostral end of this column lies the Bötzinger complex, which contains neurons that inhibit other medullary neurons and phrenic motor neurons during expiration. The Bötzinger complex roughly coincides with the so-called "rostral ventrolateral medulla" that contains vasomotor neurons (Ross et al., 1984). The rostral ventral respiratory group is found just caudal to the Bötzinger complex. This area contains many interneurons as well as premotor neurons that provide monosynaptic excitatory drive to phrenic motor neurons (Ellenberger et al., 1990). At the juncture of the Bötzinger complex and the rostral ventral respiratory group lies a group of pacemaker interneurons (the pre-Bötzinger complex) that have been hypothesized to constitute the respiratory rhythm generator (Smith et al., 1991). The caudal ventral respiratory group extends from just behind the rostral ventral respiratory group to the pyramidal decussation and contains mainly neurons that fire during expiration. As the $\mathrm{PB}$ projects to a column of neurons extending the entire length of the medulla from the facial motor nucleus to the pyramidal decussation, PB neurons can potentially form synaptic contacts with a variety of types of respiratory control neurons. Although the effects of PB stimulation on these respiratory neuronal populations has not been studied systematically, inspiratory neurons in the ventrolateral medulla have been reported to increase firing rate following electrical stimulation of the PB (Baker and Remmers, 1982). Activation of these neurons may, at least in part, underlie the respiratory facilitatory responses elicited from PB.

Apneusis occurred following glutamate injections into the rostral KF. Neurons in this area of PB project to all levels of the ventrolateral medulla, the ventrolateral and commissural subnuclei of the nucleus of the solitary tract, and the phrenic motor nucleus (Figs. 5, 7; Fulwiler and Saper, 1984; Herbert et al., 1990; Chamberlin and Saper, 1992; Portillo and Nunez-Abades, 1992). Indeed, this is the main site in the PB that projects to the nucleus of the solitary tract and the spinal cord. We found that neurons at this site that project to the ventrolateral medulla tend to innervate both its rostral and caudal extent, whereas most neurons that project to the nucleus of the solitary tract do not send collaterals to the ventrolateral medulla. Any of the descending respiratory projections of KF or all of them could have contributed to the apneustic responses we observed.

It is not likely that the KF produces apneustic responses entirely by its projection to the spinal cord, as this pathway would not be expected to increase respiratory rate. In fact, the expected reflex response to increased tidal volume would be a decrease in rate. On the other hand an excitatory spinal projection from the KF might contribute to the apneusis response by eliciting continuous phrenic activity and hence preventing complete exhalation.

Likewise the KF projection to the nucleus of the solitary tract may contribute to the production of apneusis. The role of the intermediate, interstitial, ventrolateral, and commissural subnuclei of the solitary tract (the dorsal respiratory group) in respiratory control includes the relaying of visceral sensory information such as chemoreception and pulmonary stretch to the ventral respiratory group. Furthermore, stimulation of the caudal commissural or intermediate nucleus of the solitary tract in the rat with excitatory amino acids (Bonham and McCrimmon, 1990) or electrical current (Onai et al., 1987) causes hypopnea. However, a parabrachial input to the nucleus of the solitary tract might participate in apneustic responses by disturbing the feedback signals from lung inflation, thus diminishing exhalation time and advancing the timing of the next breath.

Involvement of a direct and potent input from the rostral $\mathrm{KF}$ to the neurons in the ventral respiratory group that generate the respiratory rhythm is suggested by the sensitivity and alacrity of the apneustic response taken together with the resetting of the respiratory rhythm following application of small amounts of glutamate. Whether the rostral KF input to the ventral lateral medulla alone can cause apneusis is unclear, as it is not possible to use glutamate to selectively stimulate cells in that area without concomitant activation of the intermixed cells that project to the nucleus of the solitary tract and spinal cord. A definitive test of this hypothesis awaits discovery of a selective agonist for different cell types in the KF. Until such experiments are done, the possibility remains that the $\mathrm{PB}$ apneustic response relies upon activation of a combination of neuronal elements and efferent pathways from the PB.

We observed marked decreases in respiratory rate and even apnea following glutamate injection at the ventral outskirts of the Kölliker-Fuse nucleus and extending ventrally between the principal sensory and motor trigeminal nuclei. Very sensitive apneic sites were consistently found at the dorsal tip of the principal sensory trigeminal nucleus. The apneic zone contains scattered neurons that project to the ventrolateral medulla, but very few that project to the nucleus of the solitary tract or the phrenic motor nucleus (Fulwiler and Saper, 1984; Herbert et al., 1990; Chamberlin and Saper, 1992). Injections of retrograde tracers into all levels of the ventrolateral medulla labeled not only the Kölliker-Fuse nucleus neurons but also neurons located ventrally between the principal sensory and motor trigeminal nuclei, scattered among the motor trigeminal rootlets. Hence, the hypopneic effect might have been mediated by a projection to the ventral respiratory group. Such responses have been elicited by the application of excitatory amino acid receptor agonists into the Bötzinger complex, which diminishes respiratory motoneuronal activity (Bongianni et al., 1988; Koshiya et al., 1993). In addition, application of somatostatin at midlevels of the ventrolateral medulla produces apnea (Chen et al., 1990). However, the neurotransmitter used by the descending projection from the hypopneic site adjacent to the KF is not known.

The finding that glutamate microstimulation in the area adjacent to the PB can cause apnea is consistent with the hypothesis that the rostral pons contains an inspiratory off switch, an idea that stemmed from early observations that lesions of the PB markedly prolonged inspiratory duration causing apneustic breathing in vagotomized cats (Lumsdem, 1923). More recent experimental physiological data have also been interpreted in light of this hypothesis. For example, injections of the NMDA receptor antagonist MK-801 or the nitric oxide synthase inhibitor $N$-nitro-L-arginine into or near the $\mathrm{PB}$ prolong the duration of phrenic nerve discharge when lung inflation is witheld in paralyzed cats (Ling et al., 1992a,b). These findings suggest that activation of NMDA receptors on a population of neurons in or near the PB can terminate inspiration, although perhaps only when pulmonary stretch receptor feedback is interrupted either by cutting the vagus nerves or by withholding lung inflation. Although it is possible that the pontine apneic mechanism is disabled by pulmonary strelch receptor inputs, more likely the pontine off switch normally becomes activated more slowly than the vagally mediated cut off. If so, the prolongation of inspiratory duration after vagotomy would unmask the pontine 
mechanism. Our data are consistent with this idea, as we were able to produce apnea by glutamate injection into the pons of vagally intact animals.

\section{Functional role of $P B$ in respiration}

Although the physiological significance of the parabrachial respiratory facilitatory response is unknown, several hypotheses can be generated based upon our observations and the known connections of the PB. For example, afferents from the hypothalamus might indicate a role of the $\mathrm{PB}$ in mediating exercise hyperpnea. There is evidence for increases in breathing rate brought about by activation of the hypothalamic locomotor region (Eldridge et al., 1981). This so-called feedforward exercise hyperpnea is neurogenic in origin and independent of skeletal muscle activity. Another possibility is that the excitatory effect of $\mathrm{PB}$ stimulation on breathing might reflect a contribution of the PB to chemoreflex hyperpnea. Chemoreceptor information from the periphery could reach the $\mathrm{PB}$ via input from the nucleus of the solitary tract and central chemoreceptor data might be relayed to the $\mathrm{PB}$ from the ventrolateral medulla.

The apnea mediated by sites adjacent to the KF might play a role in airway protection. Trigeminal primary afferents innervating the upper airways and carried by the ethmoidal nerve mediate protective reflexes, including apnea and sneezing (James and Daly, 1972; Lundblad, 1984). Furthermore, primary afferent fibers in the ethmoidal nerve enter the brain just ventral to PB and innervate the Kölliker-Fuse nucleus and the lateral crescent in addition to trigeminal nuclei in muskrat (Panneton, 1991). Given the findings of Ling et al. (1992a,b) that injections of NMDA blockers into the KF region can potentiate phrenic activity (see above), it would be interesting to see if the ethmoidal nerve innervates apneic sites in the rat and if trigeminal apneic reflexes can be blocked by NMDA receptor antagonists.

Finally, the physiological role of apneusis is obscure. Prolonged inspiration might be required just before a sneeze or during some types of vocalization. However, it is also possible that the anatomical coincidence of KF cells projecting to the ventrolateral medulla, nucleus of the solitary tract, and phrenic motor nucleus might allow exogenous glutamate to elicit a coactivation of respiratory control mechanisms that does not occur naturally. A clearer picture of the possible physiological roles of the parabrachial nucleus in respiratory control should emerge upon resolution of the synaptic connectivity that underlies the respiratory responses that we observed.

\section{References}

Baker JP, Remmers JE (1982) Response of medullary respiratory neurons to rostral pontine stimulation. Resp Physiol 50:197-208.

Bassal M, Bianchi AL (1982) Inspiratory onset or termination induced by electrical stimulation of the brain. Resp Physiol 50:23-40.

Baxter DW, Olszewski J (1955) Respiratory responses evoked by electrical stimulation of pons and mesencephalon. J Neurophysiol 18 : 276-287.

Bertrand F, Hugelin A (1971) Respiratory synchronizing function of nucleus parabrachialis medialis: pneumotaxic mechanisms. J Neurophysiol 34:189-207.

Bongianni F, Fontana G, Pantaleo T (1988) Effects of electrical and chemical stimulation of the Bötzinger complex on respiratory activity in the cat. Brain Res 445:254-261.

Bonham AC, McCrimmon DR (1990) Neurones in a discrete region of the nucleus tractus solitarius are required for the Breuer-Hering reffex in rat. J Physiol (Lond) 427:261-280.

Chamberlin NL, Saper CB (1990) Physiological anatomy of the parabrachial nucleus in the rat. Soc Neurosci Abstr 16:555.
Chamberlin NL, Saper CB (1992) Topographic organization of cardiovascular responses to electrical and glutamate microstimulation of the parabrachial nucleus in the rat. J Comp Neurol 326:245-262.

Chen Z, Hedner T, Hedner J (1990) Local application of somatostatin in the rat ventrolateral medulla induces apnea. J Appl Physiol 69: 2233-2238.

Cohen MI (1971) Switching of the respiratory phases and evoked phrenic responses produced by rostral pontine electrical stimulation. J Physiol (Lond) 217:133-158.

Fldridge FI, Millhorn DE, Waldrop TG (1981) Exercise hyperpnea and locomotion: parallel activation from the hypothalamus. Science 211:844-846.

Ellenberger HH, Feldman JL (1990) Subnuclcar organization of the lateral tegmental field of the rat: I. Nucleus ambiguus and ventral respiratory group. J Comp Neurol 294:202-211.

Ellenberger HH, Feldman JL, Goshgarian HG (1990) Ventral respiratory group projections to phrenic motoneurons: electron microscopic evidence for monosynaptic connections. J Comp Neurol 302: 707-714.

Feldman JL (1986) Neurophysiology of breathing in mammals. In: Handbook of physiology, Sect 1, Vol 4, pp 463-524. Bethesda, MD: American Physiological Society.

Fournier M, Feldman JL (1988) Microinjections of excitatory amino acids into the region of the Koelliker-Fuse nucleus alter phrenic motoneuronal activity in the rat. Soc Neurosci Abstr 14:183.

Fulwiler CE, Saper CB (1984) Subnuclear organization of the efferent connections of the parabrachial nucleus in the rat. Brain Res Rev 7:229-259.

Harper RM, Frysinger RC, Trelease RB, Marks JD (1984) State-dependent alteration of respiratory cycle timing by stimulation of the central nucleus of the amygdala. Brain Res 306:1-8.

Herbert H, Moga MM, Saper CB (1990) Connections of the parabrachial nucleus with the nucleus of the solitary tract and the medullary reticular formation in the rat. J Comp Neurol 293:540-580.

James JE, Daly M de B (1972) Reflex respiratory and cardiovascular effects of stimulation of receptors in the nose of the dog. J Physiol (Lond) 220:673-696.

Koshiya N, Huangfu D, Guyenet PG (1993) Ventrolateral medulla and sympathetic chemoreflex in the rat. Brain Res 609:174-184.

Ling L, Karius DR, Speck DF (1992a) Role of NMDA receptors in the pontine pncumotaxic mcchanism in the cat. FASEB J 6:A1825.

Ling L, Karius DR, Fiscus RR, Speck DF (1992b) Endogenous nitric oxide required for an integrative respiratory function in the cat brain. J Neurophysiol 68:1910-1912.

Lipski J, Bellingham MC, West MJ, Pilowsky P (1988) Limitations of the technique of pressure microinjection of excitatory amino acids for evoking responses from localized regions of the CNS. J Neurosci Methods 26:169-179.

Lumsden T (1923) Observations on the respiratory centres in the cat. J Physiol (Lond) 57:153-160.

Lundblad L (1984) Protective reflexes and vascular effects in the nasal mucosa elicited by activation of capsaicin-sensitive substance P-immunoreactive trigeminal neurons. Acta Physiol Scand [Suppl] 529: $1-42$.

McCrimmon DR, Feldman JL, Speck DF, Ellenberger HH, Smith JC (1986) Functional heterogeneity of dorsal, ventral, and pontine respiratory groups revealed by micropharmacological techniques. In: Neurobiology of the control of breathing (von Euler C, Lagercrantz H, eds), pp 201-208. New York: Raven.

Miura M, Takayama K (1991) Circulatory and respiratory responses to glutamate stimulation of the lateral parabrachial nucleus of the cat. J Auton Nerv Syst 32:121-134.

Onai T, Saji M, Miura M (1987) Functional subdivisions of the nucleus tractus solitarii of the rat as determined by circulatory and respiratory responses to electrical stimulation of the nucleus. J Auton Nerv Syst 21:195-202.

Panneton WM (1991) Primary afferent projections from the upper respiratory tract in the muskrat. J Comp Neurol 308:51-65.

Portillo F, Nunez-Abades PA (1992) Distribution of bulbospinal neurons supplying bilateral innervation to the phrenic nucleus in the rat. Brain Res 583:349-355.

Ross CA, Ruggiero DA, Park DH, Joh TH, Sved AF, Fernandez-Pardal J, Saavedra JM, Reis DJ (1984) Tonic vasomotor control by the rostral ventrolateral medulla: effect of electrical or chemical stimulation of the area containing $\mathrm{Cl}$ adrenaline neurons on arterial pres- 
sure, heart rate, and plasma catecholamines and vasopressin. J Neurosci 4:474-494.

Smith JC, Ellenberger HH, Ballanyi K, Richter DW, Feldman JL (1991) Pre-Bötzinger complex: a brainstem region that may generate respiratory rhythm in mammals. Science $254: 726-729$.

Takayama K, Miura M (1993) Respiratory responses to microinjection of excitatory amino acid agonists in ventrolateral regions of the lateral parabrachial nucleus in the cat. Brain Res 604:217-223. von Euler C, Trippenbach T (1976) Excitability changes of the inspiratory "off-switch" mechanism tested by electrical stimulation in nucleus parabrachialis in the cat. Acta Physiol Scand 97:175-188.

Wang W, Fung M-L, St John WM (1993) Pontile regulation of ventilatory activity in the adult rat. 